\title{
Andreev quantum dots for spin manipulation
}

\author{
Nikolai M. Chtchelkatchev ${ }^{1,2}$ and Yu. V. Nazarov ${ }^{3}$ \\ ${ }^{1}$ L.D. Landau Institute for Theoretical Physics, Russian Academy of Sciences, 117940 Moscow, Russia \\ ${ }^{2}$ Institute for High Pressure Physics, Russian Academy of Sciences, Troitsk 142092, Moscow Region, Russia \\ ${ }^{3}$ Department of Nanoscience and DIMES, Delft University of Technology, Lorentzweg 1, 2628 CJ Delft, the Netherlands
}

(Dated: October 28, 2018)

\begin{abstract}
We investigate the feasibility of manipulating individual spin in a superconducting junction where Bogolyubov quasiparticles can be trapped in discrete Andreev levels. We call this system Andreev Quantum Dot (AQD) to be contrasted with a common semiconductor quantum dot. We show that AQD can be brought into a spin-1/2 state. The coupling between spin and superconducting current facilitate manipulation and measurement of this state. We demonstrate that one can operate two inductively coupled AQD's as a XOR gate, this enables quantum computing applications.

PACS numbers: 05.60.Gg,03.67.-a,73.21.-b
\end{abstract}

Manipulation and operation of individual quantum systems and arrays of such systems, so-called "quantum machines" is now in focus of both experimental and theoretical research 1, 2]. The progress in quantum computing algorithms [3] has demonstrated potential applicability of quantum mechanics thus stimulating various proposals to implement arrays of operational two-state systems (qubits) in solid-state [4, 5, 6, 7, 8, 9, 10, 11, 12, 13]. Many proposals concern quantum dots. The quantum dots are often referred to as artificial atoms since they confine a discrete number of particles that occupy discrete quantum states. In contrast to atoms, the properties of quantum dots can be tuned and their charge and spin degrees of freedom can be controlled. This would allow for quantum manipulation. An interesting and elaborated proposal [4, 5] utilizes spin states of semiconductor quantum dots. However, the complexity of the manipulation schemes proposed and severe difficulties with the read-out of these spin states [5, 14] drives one to think of alternatives.

Below we present an alternative scheme for individual spin manipulation. We concentrate on sufficiently resistive superconducting constrictions where individual Bogolybov quasiparticles can be trapped in discrete Andreev bound states. We refer to such system as Andreev Quantum Dots (AQD). An AQD resembles a common quantum dot as long as discreetness of a (quaisi)particle number, spectrum and spin is concerned. Albeit in contrast to a common quantum dot the charge of the AQD is not fixed. This allows for superconducting current in the constriction and makes electron-electron interaction negligible.

We propose to utilize spin states of the AQD's. We show that an AQD can be brought to the state with spin$1 / 2$ that persist over a long time. It is important that the spin direction in this state determines the superconducting current in the constriction, thus solving the read-out problem. We demonstrate that the spin state of a single AQD can be manipulated. Further, the two dots can be inductively coupled to make a XOR quantum gate.
Quantum information theory [3] proves that this enables one to build a universal quantum computer.

The AQD can be formed in any constriction between two superconducting leads that have a gap in energy spectrum. If an electron with the energy below the gap tries to escape to the bulk of a superconductor, it is reflected back as a hole (Andreev reflection [15]), which also can not escape due to the same reason. So that, the junction confines quasiparticles that are coherent mixtures of electron and holes. Their discrete energy levels and eigenfunctions are determined by Bogoliubov equations $(\mathrm{BdG})[16]$. It is sometimes forgotten that these equations do possess a spin structure. Bogoliubov eigenfunctions are made of two spinors 17] $u^{\alpha}, v^{\alpha}$ [coefficients of the Bogoliubov transformation 16] $\left.\Psi(r, \sigma)=\sum_{n}\left(u_{n}(r, \sigma) \gamma_{n}+g^{\sigma \mu} v_{n}^{*}(r, \mu) \gamma_{n}^{\dagger}\right)\right]$ that satisfy

$$
\begin{aligned}
& \varepsilon u^{\alpha}=\hat{H}_{\beta}^{\alpha} u^{\beta}+\hat{\Delta} v^{\alpha} \\
& \varepsilon v^{\alpha}=-\left[\hat{H}^{*}\right]_{\beta}^{\alpha} v^{\beta}+\hat{\Delta}^{*} u^{\alpha},
\end{aligned}
$$

Here "hat" denotes an operator over orbital degrees of freedom. We make explicit the spin structure of the single-particle Hamiltonian $H$ and pair potential $\Delta$, $g_{\alpha \beta} \equiv i \sigma^{y}$ being metric tensor in spinor space [17, $\left(\hat{H}^{*}\right)_{\beta}^{\alpha} \equiv g^{\nu \alpha}\left(\hat{H}_{\mu}^{\nu}\right)^{*} g_{\mu \beta}$. By virtue of Eq. (1) quasiparticle energy levels always come in pairs: each eigenstate with energy $\varepsilon$ has a counterpart with energy $-\varepsilon$. This is due to a double-counting: there are two quasiparticle eigenfunctions per each state of $H$. Should $\hat{H}$ possess no spin structure, Andreev levels are spin-degenerate. For many problems that do not involve spin, one can avoid the double counting by considering one non-degenerate level. This technical trick does not correspond to original formulation of superconductivity theory [16, 18], neither it gives the correct description of spin in superconductors.

We concentrate on a short constriction, such that the typical time for an electron $\tau_{\text {flight }}$ to traverse the junction satisfies the condition $\tau_{\text {flight }} \ll \hbar / \Delta$. In the limit $\tau_{\text {flight }} \Delta / \hbar \rightarrow 0$, and in the absence of magnetic field Andreev levels are spin-degenerate and can be universally 
expressed [19] through eigenvalues $T_{n}$ of the transmission matrix square, $\varepsilon_{n_{1}, n_{2} ; \sigma}=\operatorname{sign}\left(n_{2}\right) \Delta \sqrt{1-T_{n_{1}} \sin ^{2}(\varphi / 2)}$. Here the integer index $n_{1}$ labels orbital channels, $n_{2}=$ $\pm 1, \sigma= \pm 1$ is spin-index and $\varphi$ stands for the superconducting phase difference between the leads.

Andreev levels that are relevant for electron transport, and for manipulation of spin states, originate from $T_{n} \lesssim 1$. These levels are distributed in energy strip $\Delta|\cos (\varphi / 2)|<|\varepsilon|<\Delta$. Their typical spacing is given by $\delta E \sim \Delta G_{Q} R, R$ being the normal state resistance of the constriction, $G_{Q}$ being the conductance quantum [20]. This estimation is valid for any sufficiently disordered constriction except tunnel junctions for which all $T_{n} \ll 1$. To give an estimation, for $R \approx 10 \mathrm{Ohm}$ and $\Delta \approx 10 \mathrm{~K}$, the spacing $\delta E \approx 100 \mathrm{mK}$, this is typical for semiconductor QD. It is remarkable that that the Andreev levels do not depend on microscopic and geometric details of the constriction by means other than conductance and transmission amplitudes. This fact considerably simplifies the fabrication of Andreev dots with discrete Andreev levels.

In the ground state of the dot, quasiparticles occupy Andreev levels with negative energy. The $\varphi$ dependent part of the ground state energy reads $E_{0}=$ $1 / 2 \sum_{n \sigma} \varepsilon_{n \sigma} \Theta\left(-\varepsilon_{n \sigma}\right)$ where we sum over channel and spin index; $\Theta(x)=1$ for $x>0$ and zero for $x \leq$ 0 . The factor $1 / 2$ comes from the double-counting mentioned. 18] If spin splitting of Andreev levels is smaller than $\delta E$, the ground state has zero spin, since both components of the spin dublet are occupied by quasiparticles. In an excited state of the AQD, some Andreev levels with positive energy are populated. Let us concentrate on a given transport channel where there are two such Andreev levels corresponding to two spin directions. One quasiparticle fills either level, the AQD has spin-1/2. Second quasiparticle fills the level with the opposite spin resulting in an excited spin-singlet state. (Fig. 1a) The spin-1/2 state of an AQD with the lowest energy (that corresponds to the most transparent transport channel) is of particular interest because it is very stable. The transition to ground state require the $1 / 2$ change of spin. This means that a quasiparticle must either leave or enter the AQD. The probabilities of these processes contain exponentially small factors $\exp \left(-\Delta / k_{B} T\right)$, this means that at zero temperature the AQD would remain in spin- $1 / 2$ forever. The physics involved is very similar to well-known parity effect in superconducting grains 21]. Thus it is possible to preserve the system for a long time in this spin- $1 / 2$ state.

How to set the AQD to spin- $1 / 2$ state? Possibilities include microwave irradiation [22] and quasiparticle injection 23]. We concentrate on the first possibility. Let us assume that the irradiation frequency $\omega$ satisfies $\Delta+\varepsilon_{0}<\hbar \omega<2 \Delta$. Under these conditions, the absorption of the irradiation quanta takes place in the constriction only. If initial state is the ground state, each a)

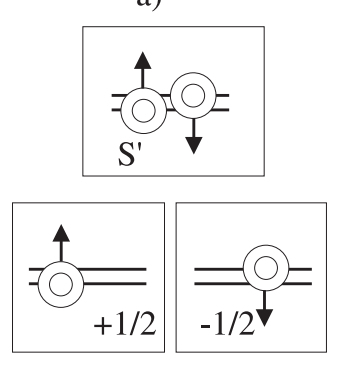

b)

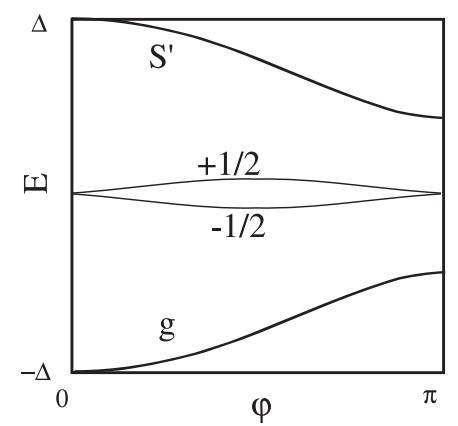

FIG. 1: a) Excited states of Andreev quantum dot in a given transport channel. b) The energies of AQD states corresponding to a given transport level versus phase difference. The lower and upper curve correspond to ground state and excited singlet respectively. The middle curves correspond to two spin-1/2 excited states, spin splitting being due to spinorbit interaction.

absorption generates two quasiparticles. There are two possibilities: 1) both quasiparticles appear in bound Andreev states; 2) one quasiparticle appears in a bound state whereas another one acquires energy $>\Delta$ and gets to the extended state (photoemission); the latter quasiparticle leaves the AQD almost immediately and never comes back. Next absorption process can occur in excited state, in this case the energy balance allows extra processes: 1) two quasiparticles leave the dot, 2) one quasiparticle in a bound state is excited over the energy barrier and leaves the dot. In any case, the processes of the first type are irrelevant not changing the parity of the AQD. Processes of the second type switch the AQD between states with even and odd number of quasiparticles. If the irradiation lasts long enough for many processes of the second type to occur, the AQD is in the state with half-integer spin with $50 \%$ probability. Let us now switch off the irradiation. If there is an even number of quasiparticles in the AQD, the subsequent energy relaxation will drive the system to the ground state. For an odd number of particles, the relaxation will result in a stable single quasiparticle occupying the lowermost Andreev level, the lowermost spin-1/2 state we are after.

How to detect the spin- $1 / 2$ state? It is important that the superconducting current in the constriction is different for the states involved, since their $\varphi$-dependent energies are different. Here we concentare on a channel $n$ and count energy from the ground state energy of quasiparticles in all other channels, $E_{g}+\left|\varepsilon_{n}\right|$. The energies then are $-\left|\varepsilon_{n}\right|, 0$, and $\left|\varepsilon_{n}\right|$ for ground, spin-1/2, and excited singlet state respectively.(Fig. 1b) and the superconducting current equals to $I=e \partial_{\varphi} E / \hbar$. The change from the ground to spin- $1 / 2$ state is therefore manifested as a change of supeconducting current by a value of $\delta I \equiv e \partial_{\varphi} \varepsilon_{n \sigma} / \hbar$. The detection of such current jumps in superconducting constrictions would amount to 
the direct experimental observation of the spin- $1 / 2$ state.

How to detect $\boldsymbol{s p i n}$ in the spin- $1 / 2$ state? An important advantage of an AQD is that its spin state affects the superconducting current, the latter being detected and/or measured. This is due to spin-orbit splitting of Andreev levels. Generally, one expects such splitting given the fact that the supeconducting phase difference changes sign under time reversal so that Kramers theorem does not hold. A confusing circumstance is that the Kramers theorem does hold in the universal limit of short constriction considered. To get spin-orbit splitting, one considers extra perturbative corrections of the first order in $\tau_{\text {flight }} \Delta / \hbar$. The calculation based on the scattering matrix approach [19] with the use of Eq.(11) yields the following effective Hamiltonian for the spin-spitting in question:

$$
E_{n}^{(S O)}=\Delta\left(\boldsymbol{\alpha}_{n} \cdot \boldsymbol{\sigma}\right) \sin (\varphi)\left(\tau_{\text {flight }} \Delta / \hbar\right),
$$

$\boldsymbol{\sigma}$ being the pseudovector operator of spin. Here a dimensionless pseudovector $\boldsymbol{\alpha}_{n}$ is a property of a given Andreev level not depending on $\varphi$; it is proportional to the spin-orbit constant that routinely contains a nuclear charge of the material $Z,|\boldsymbol{\alpha}| \simeq Z\left(e^{2} / \hbar c\right)$. For example, in a quasi-ballistic SNS junction $\boldsymbol{\alpha}$ is directed along the vector product of the quasiparticle momentum $\mathbf{p}\left(|\mathbf{p}| \approx p_{F}\right)$ and the direction of the electric (crystal) field $\mathbf{E}$ for $\varphi>0$ : $\boldsymbol{\alpha} \uparrow \mathbf{p} \times \mathbf{E}$; but when $\varphi<0$, $\mathbf{p}$ changes its direction to the opposite and $\boldsymbol{\alpha} \uparrow-\mathbf{p} \times \mathbf{E}$. This change of the direction of the quasiparticle momentum (Andreev state "chirality") with the sign of $\varphi$ is the reason why the Andreev level spin-splitting in Eq.(2) has rather unusual odd dependence on $\varphi$ [this is general property of spin-orbit Andreeev level splitting]. The technical model to derive Eq.(2) was a one-channel conductor with two scatterers separated by a distance corresponding to $\tau_{\text {flight }}$. Both scattering matrices contained spinorbit part. The model showed that in not very short constrictions $\left(\tau_{\text {flight }} \Delta / \hbar \simeq 1\right)$ spin-orbit level splitting aquired more complicated dependence on $\varphi$ and could be of the order of $\Delta$ provided spin-orbit scattering was comparable with orbital one. Thus the splitting can be huge: for a material with heavy nuclei $\boldsymbol{\alpha}$ can become of the order of unity. To show how big can be the effect, let us consider an AQD embedded into a superconducting loop with self-inductance $L$. The spin produces an extra superconducting current $I_{\sigma}=e \sigma \partial_{\varphi} E^{(S O)} \hbar$ and an extra magnetic moment per spin thereby. Let us estimate the maximum possible value of this magnetic moment. For this, we set $\tau_{\text {flight }} \simeq \Delta / \hbar,|\boldsymbol{\alpha}| \simeq 1$, and concentrate on a resistive constriction $R \backsim R_{Q}$ [so that the critical current is of the order of $e \Delta / \hbar]$. The inductance $L$ of a typical SQUID loop does not exceed $1 \mathrm{~cm}[24$ thus the extra magnetic moment $\delta M \lesssim(e \Delta / \hbar) L^{2} / c \sim 10^{12} \mu_{B}$.

Let us give a simple example of spin manipulation in the AQD. For this, we need to invoke Zeeman splitting of Andreev level in magnetic field $\sigma E^{(Z)}$ in addition to spin-orbit splitting $\sigma E_{n}^{(S O)}$. This allows us to control the direction of spin quantization by the magnetic field, this possibility being absent if we work with spin-orbit only. To achieve comparable $E^{(Z)}$ and $E^{(S O)}$, the magnetic field should be almost "in plane" not affecting the flux in the SQUID loop and thus $\varphi$. Let us assume that we manage to achieve this so that the quantization axis of spin may deviate substantially from $\boldsymbol{\alpha}$. The spin wave function is therefore a coherent mixture of the states $|\uparrow\rangle$, $|\downarrow\rangle$ with spin parallel or antiparallel to $\boldsymbol{\alpha}$ respectively. Quite generally, $\Psi=a|\uparrow\rangle+b|\downarrow\rangle$, where $|a|^{2}+|b|^{2}=1$. If there is no in-plane field, the system is in the ground state $|\downarrow\rangle$,spin quantization axis being parallel to $\boldsymbol{\alpha}$. Now let us switch on the in-plane field. The Hamiltonian governing dynamics of the wave function will thus become : $H=g \mu_{B}(\boldsymbol{\sigma} \cdot \mathbf{H})+\hat{E}_{(S O)}$. Let us assume the simplest form of the resulting Hamiltonian: $H=E^{(Z)} \hat{\sigma}_{y}+E^{(S O)} \hat{\sigma}_{z}$, $E^{(Z)} \sim E^{(S O)}$. The wave function will then evolve according to

$$
\begin{array}{r}
\Psi(t)=|\downarrow\rangle\left(\cos (\Omega t)+i \sin (\Omega t)\left(E^{(S O)} / \hbar \Omega\right)\right)- \\
-|\uparrow\rangle \sin (\Omega t)\left(E^{(Z)} / \hbar \Omega\right),
\end{array}
$$

where $\hbar \Omega=\sqrt{\left(E^{(S O)}\right)^{2}+\left(E^{(Z)}\right)^{2}}$ is the frequency of Rabi oscillations. It is important to note that these oscillations can be readily detected since they produce an alternating current

$$
I_{a}(t)=2 \sin ^{2}(\Omega t)\left(\frac{E^{(Z)}}{\hbar \Omega}\right)^{2} e \partial_{\varphi} E^{(S O)} / \hbar .
$$

Another way of manipulation is readily borrowed from the quantum optics: if the in-plane magnetic field oscillates with the resonant frequency $E_{S O}$, a significant manipulaton effect can be achieved even if $E^{(Z)} \ll E^{(S O)}$ 25]. A general unitary transformation of the spin-wave function can be performed exposing the junction to the time-dependent $E^{(Z)}$, quite similar to many other solidstate implementations of the qubits.

Let us discuss now how the Andreev quantum dots can be utilized for universal quantum computations. An AQD in the spin- $1 / 2$ state would be a qubit. We have discussed above how to manipulate the spin of a single AQD. This is how the single-qubit operations can be performed. A quantum computation algorithm should involve twoqubit operations as well. An important theoretical result [3] establishes that the XOR operation with two qubits along with single-qubit operations forms a minimum set that is sufficient to build up an arbitrary complex universal quantum computer. The XOR operation does the following: given two qubits in the states $|x\rangle,|y\rangle$, it leaves the $|y\rangle$ state unchanged if $|x\rangle=|\uparrow\rangle$, while flipping it when $|x\rangle=|\downarrow\rangle$. So we concentrate on a possible realization of XOR operation for two AQD's.

The basic idea is to organize the interaction between AQD's via inductive coupling between SQUID loops containing these AQD's [see Fig. 2. The two-qubit opera- 


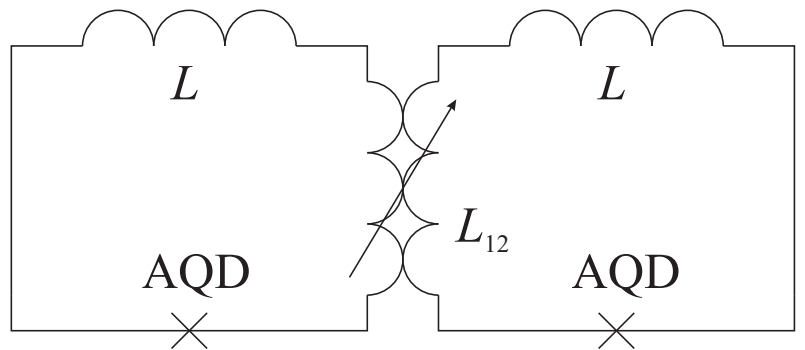

FIG. 2: The interaction between AQD qubits is organized by means of variable inductive coupling. This allows for further arraying. The circuit shown can be used as an XOR gate.

tions are performed by varying the mutual inductance between two given loops. Indeed, in this case the interaction between two AQD's can be described by a simple hamiltonian $H=L_{12} I_{1} I_{2}, L_{12}$ being the mutual inductance, $I_{1(2)}$ standing for operators of currents in corresponding loops. Owing to spin-orbit interaction, each current depends on spin state of corresponding AQD: $I_{1,2}=I_{1,2}^{(0)}+I_{1,2}^{s} s_{1,2}^{z}$. Here we choose $z$-axis in the space of each spin in the direction of corresponding pseudovector $\boldsymbol{\alpha}$. This is to stress the following circumstance: although the pseudovectors $\boldsymbol{\alpha}$ in different AQD's may differ, there is always only one component of spin that is reflected in the current and therefore takes part in the interaction. So that, the relevant part of the Hamiltonian can be written as

$$
H=H_{1} s_{1}^{z}+H_{2} s_{2}^{z}+H_{12} s_{1}^{z} s_{2}^{z} .
$$

This simple Ising-type form of Hamiltonian brings us to the old-fashioned but solid "optical" quantum computer [26]. In this approach, the one-bit operations are performed at $H_{12}=0$ by pulses at resonant frequencies $H_{1} / \hbar$ or $H_{2} / \hbar$, the pulse duration being tuned to achieve the spin flip. The XOR operation is performed at $H_{12} \neq 0$ by the same pulse with frequency $\left(H_{1}+H_{2}\right) / \hbar$. An alternative way is to use non-oscillating pulses of $H_{12} / \hbar$. Such pulses would shift phases of two states with antiparallel spins with respect to the phases of the states with parallel spins thus realizing "quantum phase shift gate" [27]. The XOR operation can be performed by combining two such phase shifts with two rotations of the target spin.

This approach of organizing two-qubit interactions has two important practical advantages. First, in contrast to other spin-based proposals, the interaction does not have to be organized at microscopic level. To exaggerate, one can use inch-scale transformers to vary inductive coupling between the AQD's. To make a practical suggestion, one can use the well-developed techniques of SQUID circuitry [28, 29] to couple, array, bias, and measure many AQD qubits. Second advantage is the simple Ising form of the resulting interaction that prevents undesired phase shifts and simplifies design of complicated quantum circuits.

To conclude, we analyze prospectives of Andreev quantum dots for spin manipulation and quantum computing. Our theoretical results seem to be promising enough to launch detailed experimental investigations and design efforts in this direction.

We wish to thank RFBR (projects No. 03-02-06259, 03-02-16677, and 03-02-17494), the Netherlands Organization for Scientific Research (NWO), the Swiss NSF, the programs of the Russian Ministry of Science: Mesoscopic systems and Quantum computations and the program of the leading scientific schools support.

[1] D. Vion et al., Science 296, 886 (2002)

[2] Y. Yu et al., Science 296, 889 (2002)

[3] A. Ekert, R. Jozsa, Rev. Mod. Phys. 68, 733 (1996).

[4] D.Loss, D.P. DiVincenzo, Phys. Rev. A 57, 120 (1998).

[5] P. Recher, D. Loss, J. Levy, cond-mat/0009270

[6] A. Shnirman et al., Phys. Rev. Lett. 79, 2371 (1997)

[7] D.V. Averin, Solid State Commun. 105, 659 (1998).

[8] J.E. Mooij et al., Science 285, 1036 (1999).

[9] B.E. Kane, Nature 393, 133 (1998).

[10] Yu. Makhlin et al., Rev. Mod. Phys. 73, 357 (2001).

[11] L.B. Ioffe et al., Nature 398, 679 (1999).

[12] Y. Nakamura et al., Nature 398, 786 (1999).

[13] L.B. Ioffe et al., Nature 415, 503 (2002).

[14] P. Recher et al., Phys. Rev. Lett. 85, 1962 (2000).

[15] A.F. Andreev, Sov. Phys. JETP 19, 1228 (1964)

[16] P.G. de Gennes, "Superconductivity of Metals and Alloys", chapters 5, 8, W.A. Benjamin, inc., New York Amsterdam 1966.

[17] L.D. Landau and E.M. Lifshitz, in Quantum Mechanics, Course in Theoretical Physics Vol. 3 (Pergamon Press, Oxford, 1977).

[18] Bardeen et al., Phys. Rev. 187, 556 (1969). Communications with our colleagues have convinced us that this basic point has to be discussed in this article.

[19] C.W.J. Beenakker, Phys. Rev. Lett. 67, 3836 (1991).

[20] Y. Imry, Introduction to mesoscopic physics, Oxford University Press, 1997.

[21] D.V. Averin et al., Phys. Rev. Lett. 69, 1993 (1992); M.T. Tuominen et al., Phys. Rev. Lett. 69, 1997 (1992).

[22] N.I. Lundin et al., Superlattices and Microstructures 25, 937 (1999)

[23] G. Wendin et al., Superlattices and Microstructures 25, 983 (1999).

[24] K. K. Likharev, Dynamics of Josephson Junctions and Circuits (Gordon and Breach Science Publishers, Amsterdam, 1991).

[25] L. Mandel and E. Wolf, Optical Coherence and Quantum Optics, (Cambridge Univ. Press, Cambridge, UK, 1995).

[26] S.A. Lloyd, Science 261, 1569 (1993).

[27] A. Barenco et al., Phys. Rev. Lett. 74, 4083 (1995)

[28] Q.A. Turchette et al., Phys. Rev. Lett. 75, 4710 (1995)

[29] O.A. Mukhanov et al., Physica C 368, 196 (2002) 